\title{
El concepto de contacto articular alternativo de la rodilla
}

\section{RESUMEN}

La observación radioscópica de la interlínea articular de rodillas de cadáveres sin anomalías anatómicas durante movimientos de abducción y adducción, realizada en estudios previos del autor, permitió apreciar la aparición de pérdida de contacto entre las superficies articulares mediales o laterales respectivamente. El presente artículo tuvo como objetivo rememorar dichos estudios y documentar las apreciaciones visuales mediante el análisis de neumoartrografías seriadas obtenidas durante movimientos mediolaterales de la rodilla. La realización de mediciones sobre las imágenes radiográficas y su tratamiento estadístico confirmaron los hallazgos obervacionales, poniendo de manifiesto que la separación de las superficies articulares contralaterales constituye un fenómeno asociado a los movimientos mediales ó laterales de la rodilla que sólo permite la presencia de contacto simultáneo en ambos compartimentos en posiciones intermedias entre la abducción y la adducción. La evaluación de éstos resultados en relación con hallazgos en seres humanos vivos y con resultados obtenidos por otros autores sugiere que en el transcurso de su función normal la rodilla puede establecer contacto indistintamente en cualquiera de los compartimentos, medial o latera, o en ambos simultaneamente. Este concepto «contacto articular alternativo» puede abrir nuevas vías para la investigación y conocimiento de aspectos oscuros o poco conocidos del funcionamiento de la articulación.

Palabra clave: Biomecánica, rodilla, cinemática

\section{SUMMARY}

The radioscopical observation of the interline joint on knees of corpses with no anathomical anomalities during the abduction and adduction movements, shown in previous studies by the author, permitted to see the apparition of contact lose in between the joint surfaces both medium and lateral. The present article had as object to review the mentioned studies and to document the visual apreciations by means of the neumoartrographic analysis series obtained during mediumlateral knee movements. The realisation of measurements on the radiographical images and there statistic treatment confirmed the viewing findings, manifesting that the contralateral joint surface separation is a associated phenomena to the medium or lateral movements of the knee that only permits the presence of simulataneus contact in both comparments in middle positions between the abduction and the adduction. The evaluation of this results in relation with the findings in human livings and with results obtained by other authors suggest that during the normal function the knee can establish indistinct contact in any of the comparments, medium or lateral, or in both simultaneusly. This concept of "alternative joint contact" can open new ways to investigation and knowledge of darker aspects or not well known of the joint function.

\section{Key words: Biomechanic, knee, kinematics.}

\section{INTRODUCCIÓN}

Se ha afirmado que la articulaciónde la rodilla debe de conciliar los imperativos contradictorios: poseer una gran estabilidad y alcanzar una gran movilidad. Parecería que para dar satisfacción los autores clásicos adjudicaron la movilidad al plano sagital y la estabilidad al plano frontal, dejando al plano transverso en una situación intermedia. Acreditados tratadistas como Steindler o Kapandji describieron la rodilla como un mecanismo de dos grados de libertad de movimiento; uno "principal», la flexión-extensión y otro «accesorio», la rotación sobre el eje longitudinal de la pierna. De acuerdo con estas premisas, en sus libros, los movimientos laterales fueron excluídos de los capítulos dedicados a la descripción de la movilidad de la rodilla. Las sumarias referencias a los mismos -en términos tales como «una ligera amplitud abductora y adductora es natural para ciertas constituciones, pero cualquier grado acusado es patológico» Steindler ${ }^{31}$, o bien «cierta holgura mecánica con la rodilla en flexión permite movimientos de lateralidad....» Kapandji ${ }^{12}$ se encuentran incluídos bajo los epígrafes dedicados a la «Función de los Ligamentos» y a la «Estabilidad transversal» respectivamente.

Sin embargo, años atrás realizamos un trabajo experimental destinado a estudiar los movimientos mediolaterales de rodillas cadavéricas, con el fin de determinar su amplitud, sus centros instantáneos de rotación y los desplazamientos asociados de las superficies articulares durante el cuál, al igual que otros autores ${ }^{2,9,10,11,13,18}$ observamos que la rodilla posee un rango significativo de movilidad mediolateral. También se observó que desviaciones angulares de la tibia de magnitud fisiológica se acompañaron de variaciones de amplitud de la interlínea articular radiológica con aparición de fenómenos de vacío; concluyendo que la adducción implica la pérdida de contacto con separación progresiva de las superficies articulares en el compartimento latera; mientras que la abducción se asocia a una separación articular 
media. ${ }^{26,27,28}$ Estas aseveraciones entraron en contradicción con concepciones previas muy arraigadas acerca de la función de la rodilla.

La mayor parte de las estimaciones publicadas de la amplitud normal de la movilidad lateral de la rodilla fueron realizadas con ocasión de estudios sobre fracturas articulares, lesiones ligamentosas ${ }^{9}, 10,11,13,18,20,22 \mathrm{u}$ osteotomías tibiales para el tratameinto de la gonartrosis ${ }^{1,6}$. Estas coincidencias inducen a asociar movilidad lateral con patología y desdeñar su posible significación físiológica. Por otra parte, aunque la pérdida de contacto entre las superficies articulares constituye una constatación rutinaria en las exploraciones artroscópicas y neumoatrográficas, suele interpretarse como una consecuencia de la aplicación de tensiones mediolaterales no físiológicas y no como un comportamiento natural de la articulación. La presencia de movimientos de abducción-adducción de la rodilla durante la marcha normal fue demostrada por varios autores hace ya varias décadas $5,14,16$; pero estos movimientos fueron atribuídos a la deformabilidad del cartílago articular o interpretados como un movimiento asociado a la flexión, como consecuencia de la diferente geometría de ambos cóndilos femorales ${ }^{17}$.

Frente a la raigambre de éstas concepciones los argumentos en favor de la pérdida de contacto articular contralateral durante los movimientos fisiológicos de abducción-adducción de la rodilla -basados en la observación visual de la articulación en la pantalla de un monitor radiológico- poseían escasa capacidad persuasiva.

Por esta razón se recuperan los registros radiográficos de nuestras experiencias en búsqueda de pruebas objetivas del contacto monocompartimental observado en asociación a movimientos laterales de la rodilla de magnitud fisiológica y se realizaron nuevas experiencias en seres humanos vivos. En el presente artículo se rememoran los hallazgos observacionales; se describen los métodos y los resultados de la búsqueda realizada y se discuten sus repercusiones.

\section{MATERIAL Y MÉTODOS}

En el estudio original se utilizaron 10 especímenes frescos extraídos de cadáveres, cuyos fémures fueron atomillados a una plataforma de madera que se fijó solidamente sobre una mesa radiográfica quedando la rodilla en posición frontal. La tibia, mantenida en $10^{\circ}$ de flexión se unió a un dispositivo que solo permitió libremente rotaciones y traslaciones en el plano frontal. Un sistema de tracción a tornillo accionado por una manivela permitió desplazar gradualmente la tibia en dirección medial o lateral. A fin de determinar la posición neutra, en todos los especímenes se inyectaron intraarticularmente 10 c.c. de aire y se imprimieron movimientos mediolaterales a la tibia mientras se observaba radioscópicamente la interlínea articular a través de un intensificador de imágenes (fig. 1).

Para referenciar las distintas posiciones de la tibia y realizar su análisis cinemático se usaron placas de plomomicroperforadas las cuales, al impedir el paso de radiación, excepto a través de sus orificios, actuaron como marcadores radiológicos negativos y permitieron imprimir en una única radiografía la secuencia total del movimiento lo que condujo a una simplificación del proceso de digitalización y tratamiento de los resultados por ordenador. Como contrapartida, este perfeccionamiento metodológico impidió el registro radiográfico de las observaciones realizadas a través del monitor.

Sin embargo, en nuestras dos primeras experiencias se inyectó aire en la articulación, se utilizaron también marcadores radiológicos femorales y la tibia fue desplazada progresivamente en adducción mientras se disparaba común seriador angioradiográfico una secuencia de radiografías con intervalos de $200 \mathrm{msg}$. Se obtuvieron así series de imágenes neumoartrográficas de las rodillas en grados crecientes de desplazamiento angular, cuantificable a través de las variaciones en la posición de los marcadores radiográficos ligados a la tibia, en las que pudieron identificarse claramente los límites del hueso subcondral, del cartílago articular y de los menis$\cos$ (fig 2). Dichas series fueron recuperadas y sirvieron de base al estudio actual.

Las radiografías de cada serie fueron proyectadas y se obtuvieron ampliaciones, sobre las que se realizaron las siguientes mediciones:

1) ángulo fémur-tibia, formado por dos rectas que unen respectivamente dos marcadores femorales y tibiales homólogos (fig 3),

2) distancias entre las placas epifisarias femorales y tibiales medial y lateral, correspondientes a la amplitud total de la interlínea articular radiológica y

3) distancia entre las superficies de los cartílagos articulares fermorales y tibiales medial o lateral o espacio intercartilaginoso (fig 4). Se determinó el ángulo girado por la tibia hallando las diferencias entre el valor del ángulo fémur-tibia inicial y el correspondiente a cada nueva posición (fig 3). Las distancias 2) y 3) fueron determinadas sobre las perpendiculares a la tangente común a ambos cóndilos femorales en sus puntos de tangencia medial y lateral.

Las magnitudes angulares se expresaron en grados sexagesimales y las medidas de longitud en unidades adimensionales, dividiendo su valor por el diámetro frontal de la epífisis tibial. Las variables medidas fueron sometidas a un análisis de regresión obteniéndose sus coeficientes de correlación y sus niveles de significación estadística.

\section{RESULTADOS}

La manipulación de los especímenos mostró que la tibia podía ser desplazada libremente en dirección medial o lateral dentro de cierto rango angular; superado el cuál ofreció una resistencia progresiva a cualquier lateralización adicional produciéndose un fenómeno de retroceso al suprimir la fuerza actuante. En las posiciones de desviación lateral de la tibia (abducción) se observó una línea radiolúcida interpuesta entre el cóndilo femoral y el platillo tibial internos; mientras que el compartimiento lateral mantuvo una imagen de densidad uniforme. Durante la desviación medial (adducción) se produjo el fenómeno contrario, apareciendo la línea radiolúcida en la interlínea articular lateral. 


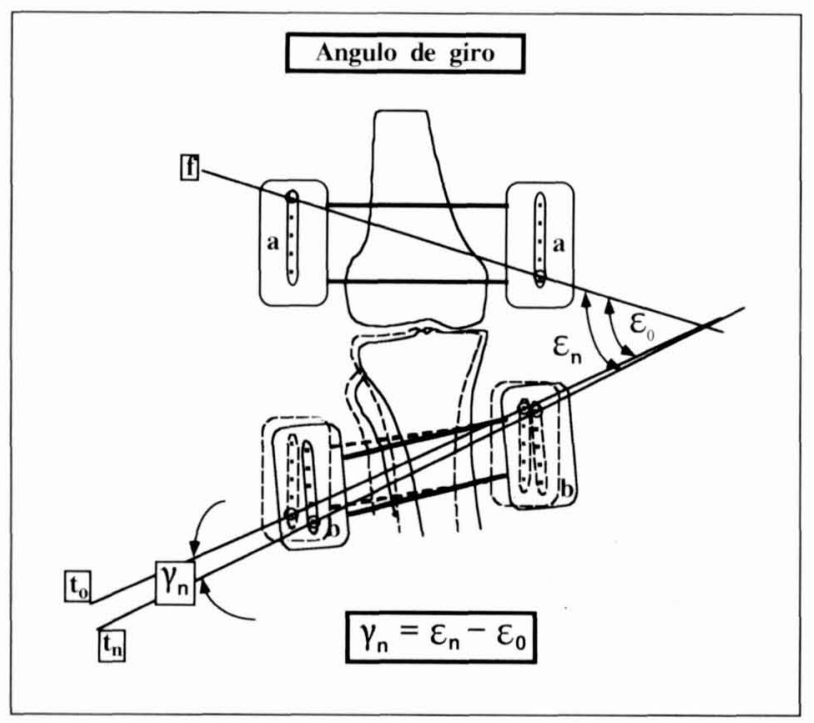

Figura 3.- La recta $f$ que une dos marcadores femorales (a) forma con la recta tn trazada entre dos marcadores tibiales (b) un ángulo (ángulo fémur-tibia, $\varepsilon_{n}$ ) diferente en cada posición. El ángulo girado por la tibia $\gamma_{n}$ para alcanzar una posición dada (n) se obtuvo restando el ángulo $\varepsilon_{n}$ del ángulo fémur-tibia en la posición inicial $\varepsilon_{0}$.

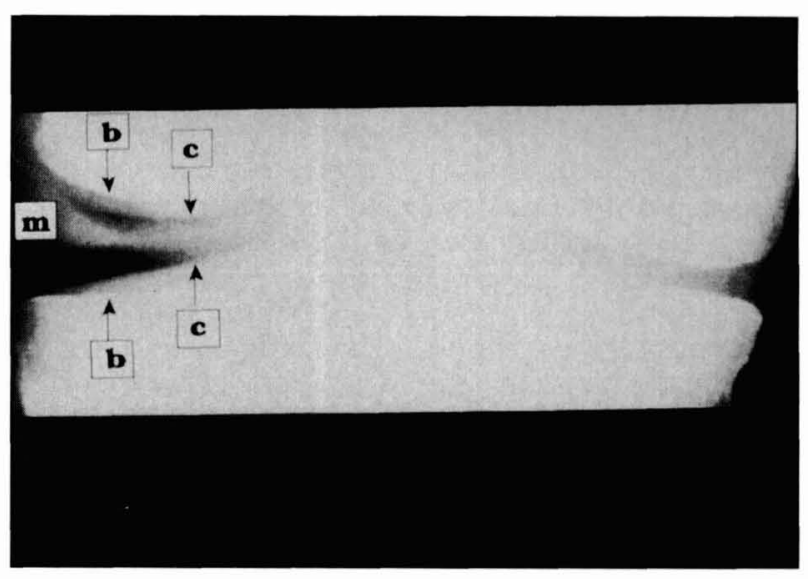

Figura 2.- Instantánea neumoartrográfica de la interlínea en acusado desplazamiento medial. Pueden observarse el menisco extermo (m) y los límites del hueso subcondral (b) y del cartílago articular (c).

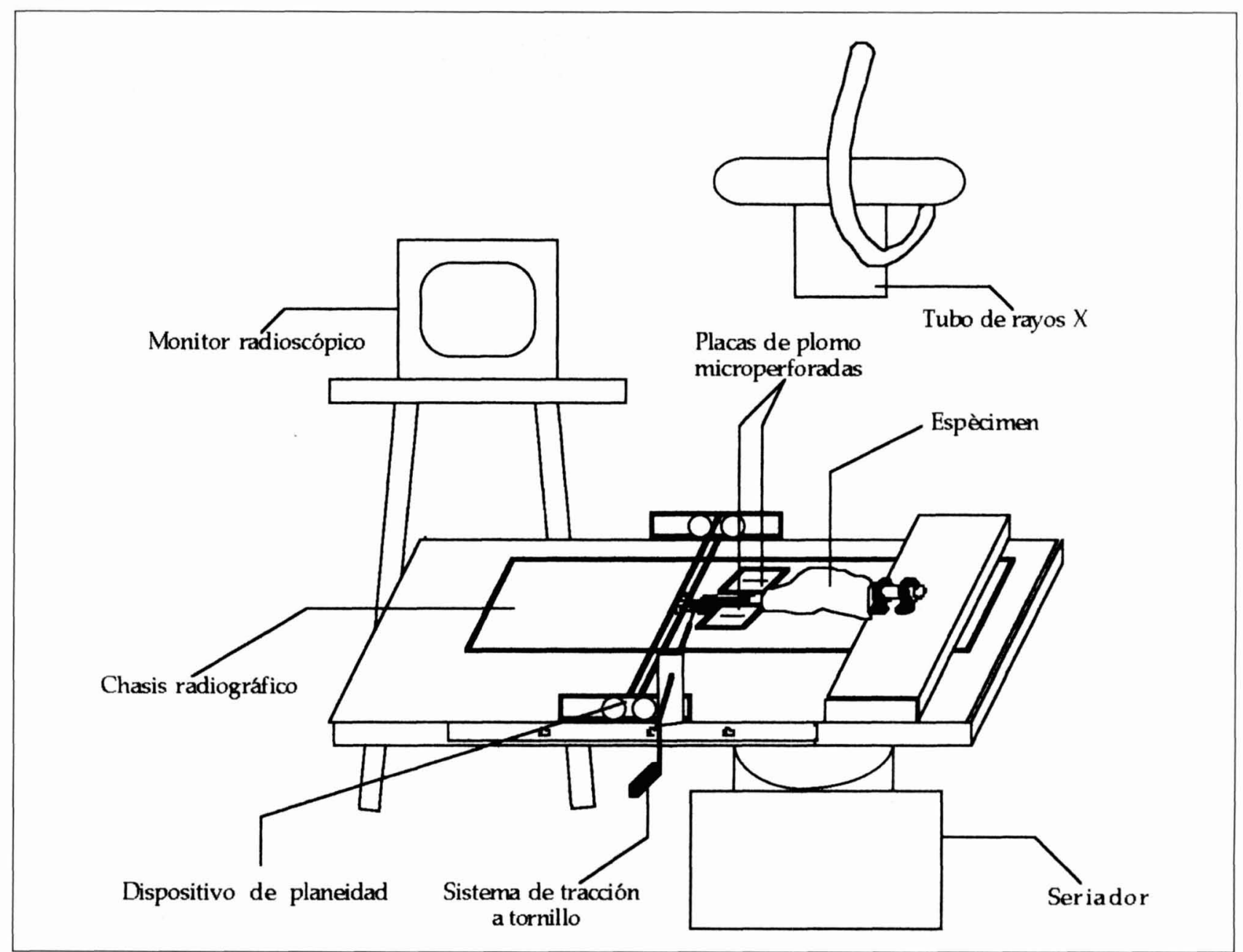

Figura1.- Representación esquemática del dispositivo experimental. Durante los movimientos mediolaterales planos, graduados mediante el sistema de tracción a tornillo, es posible registrar la trayectoria de marcadores tibiales mediante disparos radiográficos secuenciales sobre las placas de plomo microperforadas. Previa inyección de aire en la articulación el movimiento relativo de las superficies articulares puede ser observado en el monitor o registrado mediante el seriador radiográfico. 


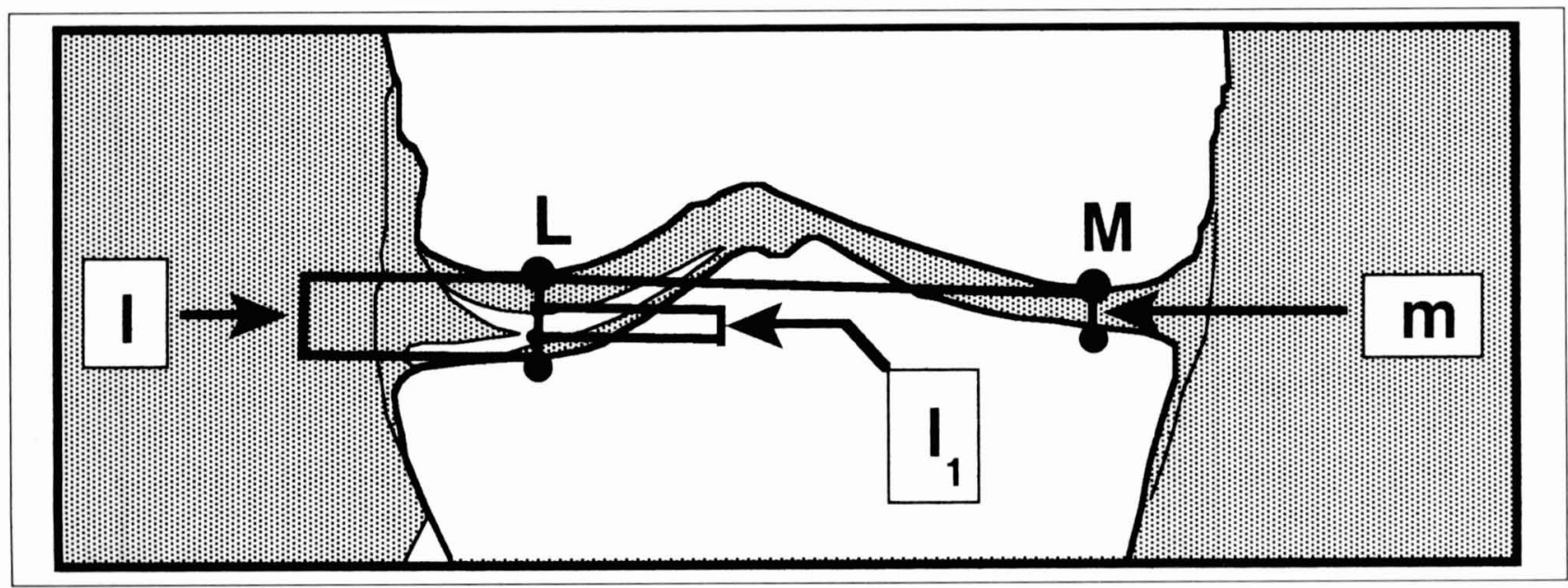

Figura 4.- Las distancias entre los contornos óseos $(\mathrm{m}, \mathrm{l})$ y entre los límites del cartílago articular $\left(\mathrm{I}_{1}\right)$ del fémur y de la tibia se midieron sobre las perpendiculares a la tangente distal a los cóndilos femorales en sus puntos de contacto medial (M) y lateral (L).

Entre ambas desviaciones se encontraron posiciones intermedias de la tibia en que ambos compartimentos mantuvieron una densidad radiológica uniforme o mostraron, en áreas limitadas de sus superficies de contacto, dos tenues líneas interpuestas simétricamente (fig 5). La presencia del margen de movilidad libre señalado al principio permitió en todos los casos situar la rodilla en la posición angular acompañada de imágenes simétricas en ambas interlíneas; posición que se mantuvo en equilibrio sin la intervención de fuerzas exteriores. Sin embargo, también se produjo equilibrio espontáneo en otras posiciones angulares acompañadas de imágenes radiolúcidas unicompartimentales.

La observación de las series neumoartrográficas evidenció que un desplazamiento angular mínimo de la posición intermedia -de magnitud inferior a $1^{\circ}$ - fué suficiente para que las imagenes radiolúcidas se insinuasen en toda la extensión de la interlínea contralateral y desapareciesen en la homolateral. El grosor de esta sombra y la distancia entre las superfícies articulares fue aumentando progresivamente conforme se incrementaba la desviación angular (fig. 6).

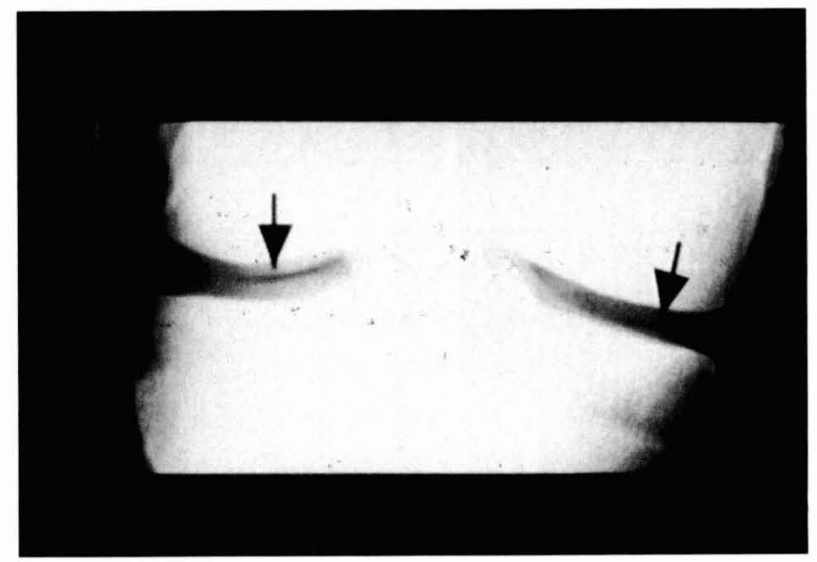

Figura 5.- Posición intermedia entre los desplazamientos medial y lateral en la que se observan dos imágenes radiolúcidas interpuestas en la parte central de ambos compartimentos; compatibles con la presencia de contacto bicompartimental periférico debido a la ausencia de carga durante la experiencia.
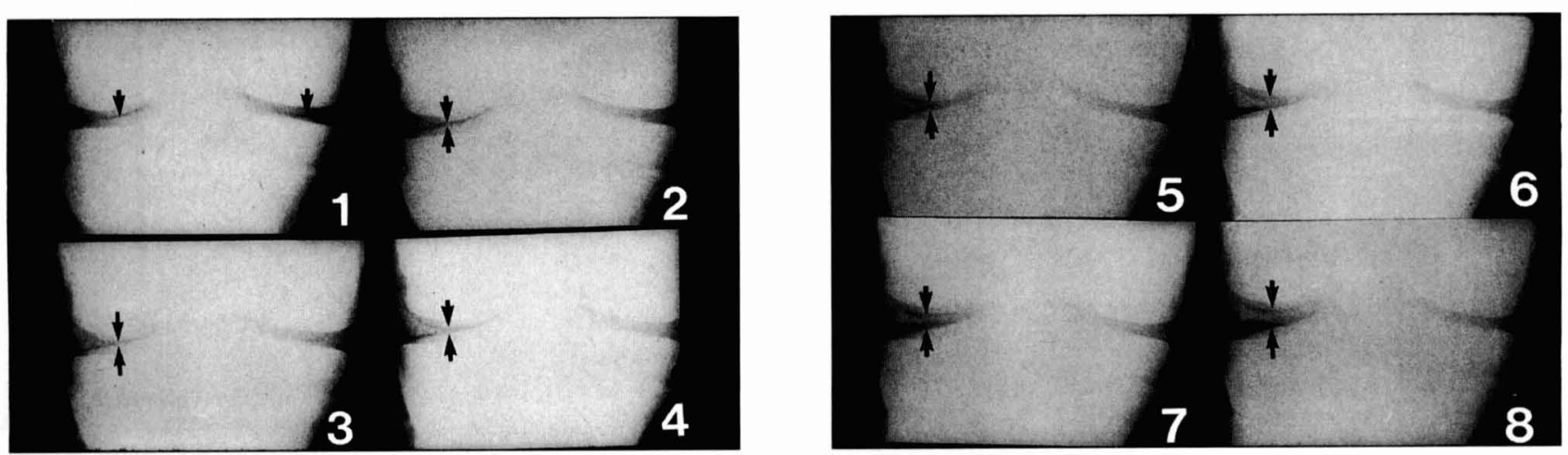

Figura 6 a y b.- Fotocomposición que muestra una secuencia de imágenes neumoartrográficas de la interlínea en grados crecientes de adducción. La imagen radiolúcida lateral ocupó la totalidad del compartimiento tras un desplazamiento mínimo (2), aumentando su espesor conforme avanzó la desviación angular, mientras que la imagen medial visible en la primera instantánea (1) desapareció. 


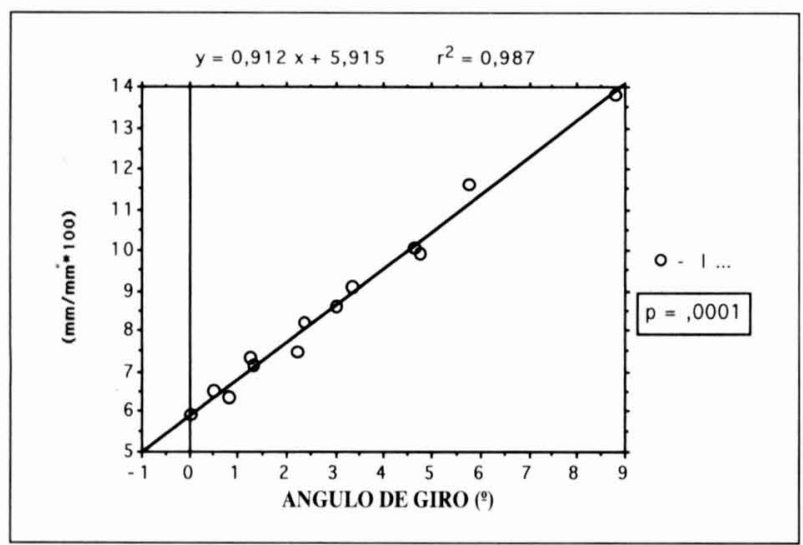

Figura 7.- Correlación lineal positiva entre la amplitud del espacio articular lateral (1, ordenadas) y el grado de desviación en adducción de la tibia (ángulo de giro, abscisas).

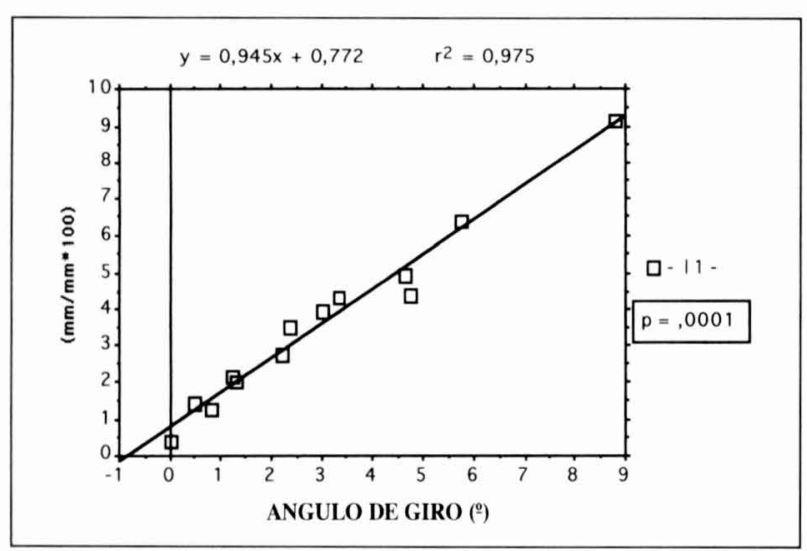

Figura 8.- Correlación lineal positiva entre la distancia intercartilaginosa ( $\mathrm{l}_{1}$, ordenadas) y el grado de adducción (ángulo de giro, abscisas).

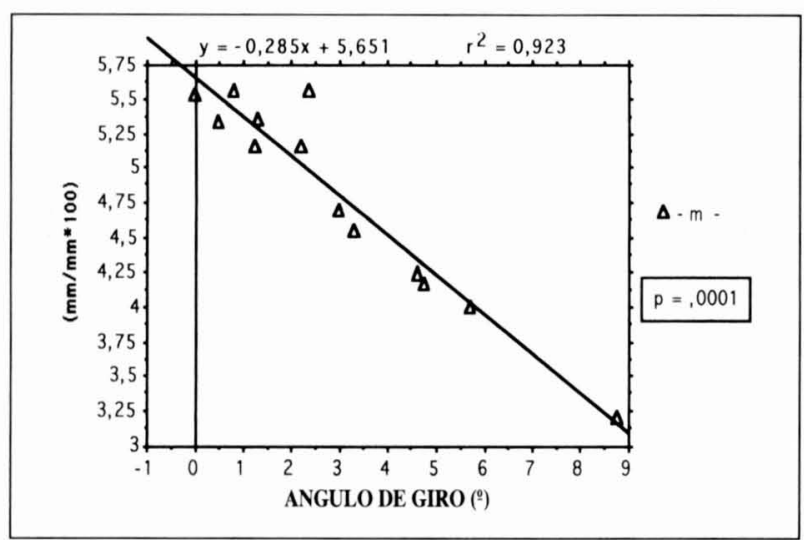

Figuira 9.- Correlación lineal negativa entre la amplitud del espacio articular medial ( $m$, abscisas) y el grado de abducción. Observese que la magnitud del estrechamiento del espacio medial $(m)$ es del orden de una tercera parte del ensanchamiento de $10 l_{1}$.
El análisis de regresión realizado demostró la existencia de correlación lineal positiva estadísticamente significativa $(\mathrm{p}<0,001)$ entre el valor del ángulo girado por la tibia y los correspondientes a la amplitud del espacio articular y de la distancia intercartilaginosa (fig. 7 y 8 ). Niveles equivalentes de correlación lineal negativa $(\mathrm{p}<0,001)$ se encontraron entre el ángulo de giro y la amplitud del espacio articular medial (fig. 9); aunque la magnitud de las variaciones de espesor del espacio articular fué inferior a la tercera parte de las correspondientes al espacio articular lateral.

\section{DISCUSIÓN}

La presencia de un rango angular dentro del cuál la rodilla puede sufrir desplazamientos sin ofrecer una resistencia apreciable parece sugerir que los ligamentos encargados de limitar los movimientos frontales permanecieron relajados o desarrollaron una tensión mínima, empezando a entrar en carga o desarrollar tensión significativa una vez superado dicho intervalo en dirección medial o lateral. Este comportamiento es compatible con el encontrado por otros autores ${ }^{10,18,25}$ pudiendo corresponderse el rango de movilidad libre encontrado en nuestras experiencias con el intervalo de menor rigidez («midrange stiffness») descrito por Markolff y $\operatorname{cols}^{18}$ en su estudio sobre rodillas cadavéricas, al que denominaron «laxitud». Sin embargo, teniendo en cuenta que todos los autores que realizaron mediciones registraron la presencia de movimientos mediolaterales en la rodilla normal in vitro e in vivo $2,6,9,10,11,18,22,25$ parecería razonable admitir que constituyen en realidad un grado de libertad de movimiento; por lo qeu al desplazamiento que ocurre entre sus límites debería de designársele como «amplitud» o «rango».

La introducción de medios de contraste en las articulaciones y la identificación de las zonas de exclusión han sido como método para determinar las superficies de contacto femorotibiales ${ }^{16,18}$. En sentido contrario, parece obvio que la presencia de contraste entre las superficies articulares presupone la ausencia de contacto entre ellas. La inyección de aire u otros gases en la rodilla como medio de contraste negativo para poner en evidencia el verdadero espacio articular y explorar sus estructuras han sido utilizado en la clínica desde hace varias décadas. ${ }^{21,30}$ Durante estas exploraciones se realizan distintas maniobras para separar las superficies articulares y permitir que el gas se insinúe entre ellas sustituyendo al líquido sinovial. Así, parece evidente que las imágenes radiotransparentes observadas en nuestras experiencias responden a la infiltración entre las superficies articulares del aire previamente inyectado; por lo que en la extensión de ésas imágenes puede afirmarse la inexistencia de contacto articular. Hipotéticamente la insuflación de aire en cantidad suficiente para producir una gran elevación de la presión intraarticular podría desencadenar una separación o distracción de las superficies articulares. Sin embargo los 10 c.c. inyectados durantes las experiencias son incapaces de generar una presión positiva significativa en la articulación lo que permite afirmar que los movimientos de abducción y adducción imprimidos a la tibia causaron la pérdida contacto entre las superficies articulares femorotibiales mediales o laterales respectivamente, mientras que su permanencia en posiciones intermedias entre ambos 
desplazamientos se acompañó de contacto simultáneo, total o periférico, en ambos compartimentos.

Se han utilizado distintos criterios para determinar la posición neutra entre la abducción y la adducción, tales como la actitud natural en bipedestación ${ }^{14}$-inaplicable a las condiciones experimentales de nuestro trabajo- o la posición adoptada espontaneamente por la rodilla en reposo, esto es, cuando no se aplican fuerzas laterales ${ }^{13}$, condición que consideramos inválida tras nuestro hallazgo de equilibrio espontáneo en distintos grados de adducción-abducción. Varios autores ${ }^{2,11,18}$ renunciaron a fijar una posición neutra ante las dificultades encontradas para determinarla con precisión. De acuerdo a nuestras observaciones, consideramos que la posición neutra debe de identificarse con la presencia de contacto articular bicompartimental. Dicha posición -coincidente con la ausencia total o la presencia simétrica de imágenes aéreas centrales en ambos compartimentos- pudo ser encontrada con facilidad durante nuestras experiencias en rodillas cadavéricas mediante manipulación de la tibia bajo control radioscópico o neumoartrográfico. Por otra parte la posición central del ligamento rotuliano sugiere que, en el vivo, la contracción del cuádriceps dará lugar a la compresión simultánea de ambos compartimentos. Con este fundamento teórico determinamos habitualmente la posición neutra en sujetos vivos, en la práctica clínica, realizando radiografías anteroposteriores de las rodillas en decúbito supino con los cuádriceps contraídos.

La presencia de variaciones en la amplitud de la interlínea articular radiológica («espacio claro» de Martin ${ }^{20}$ ) ralacionadas con los movimientos de abducción y adducción ha sido observada tanto en experiencias con articulaciones cadavéricas ${ }^{10,11,13,18,20,22}$ como en el ser humano vivo y consciente..$^{2,14}$ La posibilidad teórica de pérdida de contacto entre las superficies articulares fué predicha a partir de análisis estáticos de las fuerzas actuantes sobre la rodilla en el plano frontal ${ }^{24}$ y constituye una constatación rutinaria de las exploraciones artrográficas o artroscópicas. Refiriéndose a la fisiología de la rodilla normal, éstos fenómenos no se han relacionado entre sí hasta el momento actual; siendo atribuídas las variaciones de amplitud de la interlinea a la deformabilidaddel cartílago, las variaciones en la abducciónadducción a un movimiento asociado a la flexoexten$\operatorname{sión}^{16}$ como consecuencia de la particular geometría de los cóndilos femorales y la separación de las superficies articulares a la presencia de patología articular o a la aplicación de tensiones laterales no fisiológicas. 1,3,4,22,23 Sin embargo, de acuerdo a nuestros resultados, la causa principal de las variaciones de amplitud de la interlínea fué la separación física de las superficies articulares o su acercamiento, al triplicar aproximadamente la magnitud de la deformación conjunta del menisco y el cartílago articular. Por otra parte, la inmediata pérdida de contacto asociada con desviaciones mínimas de la posición neutra y la proporcionalidad mostrada entre el desplazamiento angular de la tibia y la separación de las superficies articulares (fig. 7 y 8) parecen acreditar que existió entre ambos fenómenos una relación de causa a efecto, de acuerdo a la cual el movimiento de la tibia en adducción dió lugar a un distanciamiento progresivo entre el cóndilo femoral y el platillo tibial lateral y el movimiento de abducción ocasionó el mismo fenómeno en el compartimento medial; mientras que el contacto bicompartimental total o periférico solo se mantuvo en la posición intermedia (fig.10).

Los fenómenos observados y descritos hasta aquí, podría atribuirse a un compartimento particular de las rodillas cadavéricas y de las condiciones de la experimentación; caracterizadas por la ausencia del peso corporal y de la contracción muscular. Sin embargo, la presencia espontánea de fenómenos de vacío observada durante el estudio de radiografías de rodillas en abducción o adducción provocada posturalmente (fig. 11) a sujetos en bipedestación asimétrica, objeto de otro artículo en éste mismo número, ponen de manifiesto que el proceso de separación articular se produce en rodillas vivas bajo condiciones de carga y no es privativo de las rodillas cadavéricas de las condiciones de la experimentación; lo cuál sugiere que debe de constituir el mecanismo normal que permite los pequeños movimientos fisológicos de lateralidad de la rodilla normal. La razón por la que este fenómeno no resulta evidente parece deberse a que la densidad radiológica del líquido sinovial que ocupa habitualmente los espacios vacíos de la articulación no permite distinguir dichos espacios de las imágenes correspondientes al cartílago articular y los meniscos. De acuerdo a estos hallazgos, parece razonable admitir que los movimientos de abducción-adducción que ocurren durante la marcha normal se acompañan de fases alternantes de contacto articular monocompartimental. Para referirnos a éste comportamiento hemos acuñado la expresión de concepto de contacto articular alternativo de la rodilla.

La concepción de la rodilla normal como una articulación que puede apoyarse indistintamente en uno u otro compartimento o en ambos simultaneamente frente a la creencia clásica de que ambos cóndilos mantienen permanentemente contacto con ambos platillos tibiales excepto en circunstancias patológicas puede implicar cambios radicales en algunos de nuestros conocimientos acerca de su funcionamiento. Así, si aceptamos éste modo de comportamiento natural deberemos de admitir la existencia de tres movimientos sagitales de la rodilla

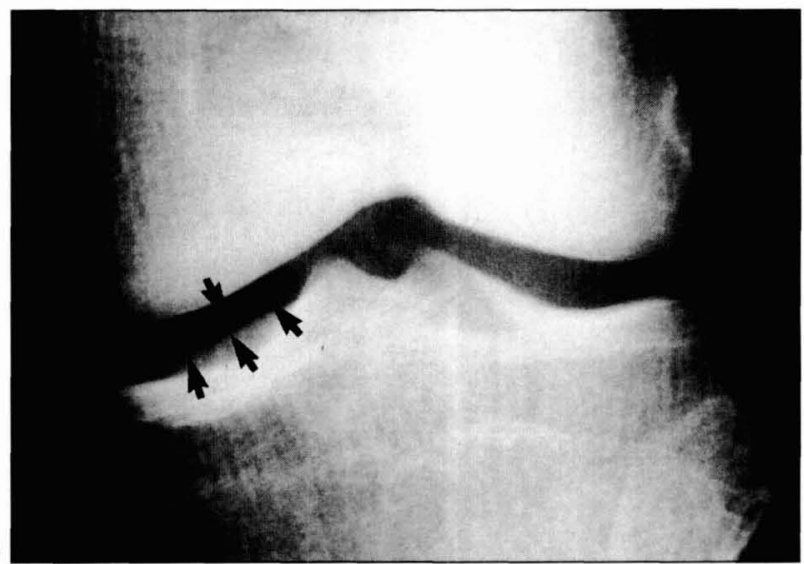

Figura 11.- Radiografía de la rodilla asintomática de un sujeto vivo tomada en abducción mantenida posturalmente durante la bipedestación asimétrica. Observese la presencia de un fenómeno de vacío -ausente de contaco articular- en el compatimento femorotibial medial. 

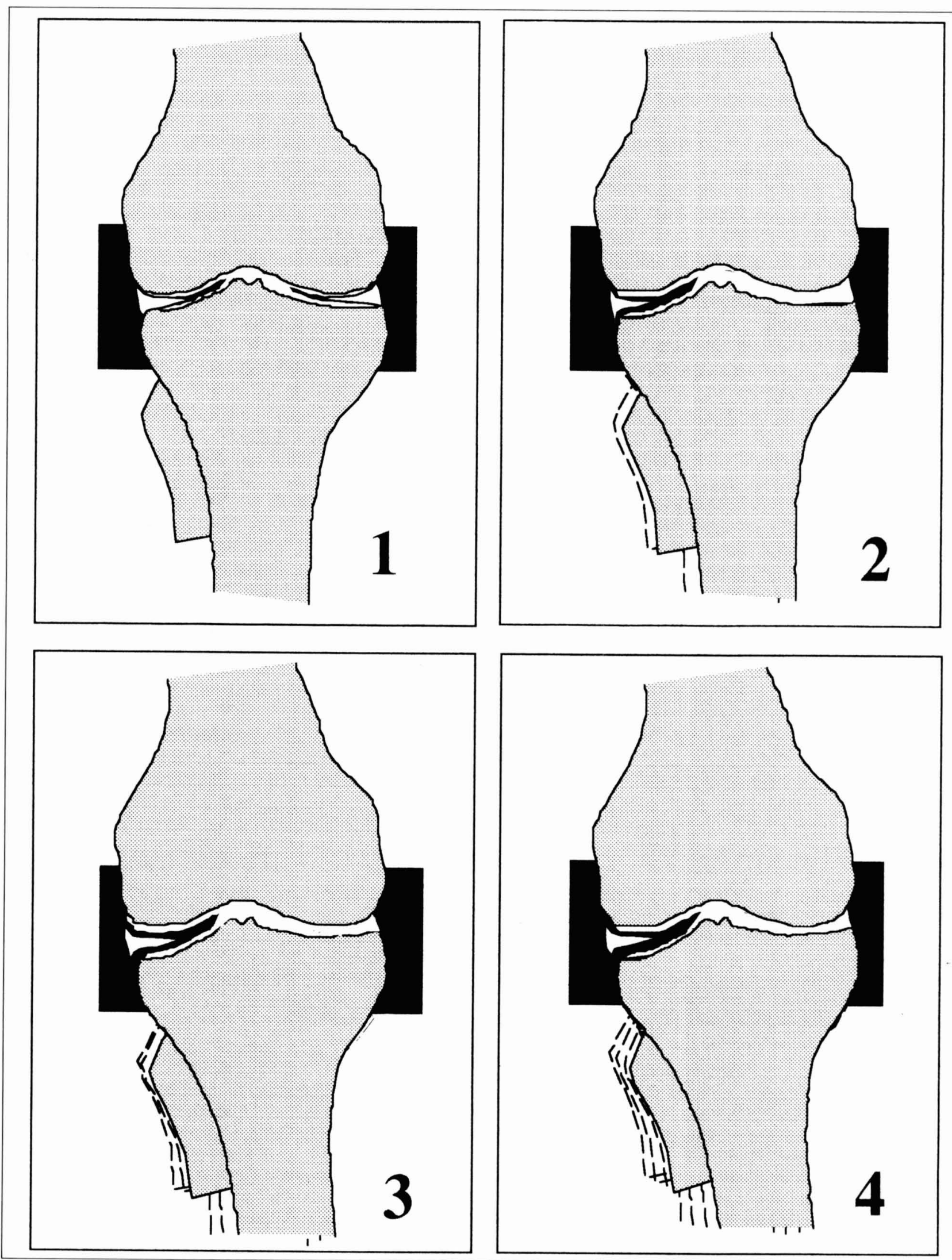

Figura 10.- Representación esquemática de los fenómenos intraarticulares asociados a los movimientos mediolaterales de la tibia. Dentro del grado de amplitud de movimiento permitido por los ligamentos la inclinación medial de la tibia (adducción) conlleva la pérdida de contacto y separación progresiva de las superficies articulares femorotibiales laterales. Durante la inclinación lateral (abducción) se observó un fenómeno similar entre las superficies articulares mediales. La situación intermedia entre los desplazamientos medial y lateral1, representa la «posición neutra". 
dependiendo del grado de abducción-adducción en que se produzcan; a los que hemos denominado flexión monocondílea lateral y flexión bicondílea. ${ }^{29}$ Por otra parte, la adducción registrada como consecuencia del momento adductor que actúa en la fase de apoyo del paso se acompañará de un contacto monocompartimental medial (fase de apoyo monocondíleo de la marcha ${ }^{26,27,28}$, cuyos mecanismos de equilibración lateral (control secuencial de la estabilidad lateral) permiten avanzar nuevas hipótesis sobre el origen muscular de la gonartrosis 27,28 . Estos y otros aspectos oscuros o mal comprendidos de la biomecánica de la rodilla han sido estudiados desde la perspectiva del contacto articular alternativo y serán objeto de próximas publicaciones.

\section{BIBLIOGRAFÍA}

1. Blaimont, P; Burnotte, J; Baillon, J.M; Duby, P.: Contribution bioméchanique a l'etude des conditions d'equilibre dans le genou normal et pathologique; Acta Orthop. Belg.; 37; 573-92; 1971.

2. Brantigan, O.C.; Voshell, A.F.: The Mechanics of the Ligaments and Menisci of the Knee Joint.; J. Bone Joint Surg.; XXIII; 44$66 ; 1941$.

3. Denham, R. A.; Bishop, R.E.D.: Mechanics of the Knee and Problems in Reconstructive Surgery; J. Bone Joint Surg.; 60-B; 345-52; 1978

4. Dugdale, T.W.; Noyes, F.R.; Styer, D.:Preoperative Plannig for High Tibial Osteotomy. The Effect of Lateral Tibiofemoral Separation and Tibiofemoral Lenght; Clin. Orthop; 274; 248-64.

5. Ebehart, H.D.; Inmman, VLT; Saunders, J.B.: Fundamental Studies of Human Locomotion and Other Informations Relating to the design of Artificial Limbs; University of California, Berkeley; 1947

6. Edholm, P; Lindahl, O; Lindholm, B; Myrnerts, R.: Olson, K.E.; Wennberg, E.: Knee Instability; Acta Orthop. Scand.; 47; 653$63 ; 1976$

7. Fuicks, D.V.; Grayson, C.D.:Vacuum pneumoatrography and the spontaneus ocurrence of gas in the joint spaces; $J$. Bone Joint Surg; 32-A; 933-38; 1950.

8. Gerson-Cohen, J.: Internal Derangements of the Knee Joint. The Diagnostic scope of the Soft Tissue Roentgen Examinations and the Vacuum Tecnique demonstration of the meniscus.; Am. J. roentgenol:; 54; 338-47; 1945.

9. Gollehon, D.L. et al.: The Role of the Posterolateral and Cruciate Ligaments in the Stability of the Human Knee; J. Bone Joint Surg.; 69-A; 233-42; 1987

10. Grood, E.S.; Noyes, F.R.; Butler, D.L.; Suntay, W.J.: Ligamentous and Capsular Restraints Preventing Medial and Lateral Laxity in intact Human Cadaver Knees. J. Bone Joint Surg.; 63-A; 1257-69; 1981 .

11. Hallén, L.G.: Lindahi, O.: Lateral Stability of the Knee Joint; Acta Orthop. Scand.; 36; 179-91; 1965.

12. Kapandji, I.A.: Cuadernos de fisiología articular; Toray-Masson, Barcelona, 1973.
13. Kennedy, J.C.; Fowler, P.J.: Medial and Aterior Instability of the Knee. An Anatomical and Clinical Study Using Stress Machines.; J. Bone Joint Surg.; 53-A; 1257-70; 1971.

14. Kettelkamp, D.B. et al.: An Electrogoniometric Study of the Knee Motion in Normal Gait; J. Bone Joint Surg.; 52-A; 775-90; 1970 .

15. Kettelkamp, D.B; Jacobs, A.V.: Tibiofemoral Contact Area. Determination and Implications; J. Bone Joint Surg.; 54-A; 349 $56 ; 1972$.

16. Kettelkamp, D.B.: Gait characteristics of the knee: normal, abnormal, and postreconstruction; AAOS. Symposium on Reconstructive surgery of the knee; C.V. Mosby Co.; 47-57; Saint Louis, 1978.

17. Maquet, P.G.J.: Biomechanics of the knee; Springer Verlag; Berlin-Heidelberg; 59-67; 1976.

18. Markolff, K.L.;Mensch, J.S.; Amstutz, H.C.: Stiffness and Laxity of the Knee. The Contribution of y the Supporting Structures; J. Bone Joint Surg; 58-A; 583-93; 1976

19. Markolff, K.L.; Mensch, J.S.; Amstutz, H.C.: Stiffness and Laxity of the Knee. The Contribution of the Supporting Structures.; J. Bone Joint Surg.; 58-A; 583-93; 1976.

20. Martin, A.F.: The Pathomechanics of the Knee Joint. I. The medial Collateral Ligament and Lateral Tibial Plateau Fractures.; J. Bone Joint Surg.; 42-A; 13-22; 1960.

21. McFraw, W.H.; Weckesser, E.C.: Pneumoarthrograms of the Knee. A Diagnosis Aid in Internal Derangements; J. Bone Joint Surg.; 27; 432-45; 1945.

22. Moore, T.M.; Meyers, M.H.; Harvey, J.P: Collateral Ligament Laxity of the Knee; J. Bone Joint Surg.; 58-A; 594-98; 1976.

23. Noyes, F.R.; Schipplein, O.D.; Andriachi, T.P.; Saddemi, S.R.; Weise, M.: The Anterior Cruciate Ligment Deficient Knee with Varus Alignement. An Analysis of gait adaptations and dinamic joint loadings; Am. J. Sports Med.; 20; 707-16; 1992.

24. O'Connor, J.; Shercliff, T.; FitzPatrick, D.; Biden, E.; Goodfellow, J.: Mechanics of the Knee; in Daniel, Akeson, O'Connor: Knee Ligaments, Structures, Function, Injury and Repair. Raven Press; Chapter 11; 201-37; New York, 1990.

25. O'Connor, J; Biden, E; Bradley, J; FiłzPatrick, D; Young, S.; Kershaw, Ch.; Daniel, D.M.; Goodfellow, J.: The MuscleStabilized Knee; in Daniel, Akeson, O'Connor: Knee Ligaments, Structure, Function, Injury and Repair. Raven Press; Chapter 12; 239-77; New York; 1990.

26. Pichel, C.M.: Aportaciones experimentales a la biomecánica de la rodilla; Libro de Resúmenes III Symposium SIB: p. 15; Barcelona, 1980.

27. Pichel, C.M; Montoya, M.A.: Pathogenesis of Osteoathritis of the Knee. New Biomechanical Hypothesis.; Rev. Rhum.; num. special juin; 0744; 1981.

28. Pichel, C.M.: «Estudio de la acción del obenque muscular externo sobre la rodilla normal y patológica»; Memoria final Proyecto Investigacion 12281/77. Fondo de Investigaciones Sanitarias (FIS). Ministerio de Sanidad; Madrid, 1982.

29. Pichel, C.M.; Matesanz, P.; López Pérez, I.; Torres, J.; Pérez Mendaña, J.; Facal, M.; Docampo, J.: Los movimientos sagitales de la rodilla: nuevo modelo cinemático; Libro de Resúmenes V Symposium SIB; La Coruña, 1982.

30. Quaintance, P.A.: Pneaumoatrography of $y$ the Knee Joint; J. Bone Joint Surg.; 20; 353-62; 1938.

31. Steindler, A.: Kinesiology of the Human Body; Charles C. Thomas; Springfield, 1955. 